\title{
Calculation of the Flory Exponent Using the Renormalization Theory
}

\author{
Xiao-Hong WANG and Ke-Lin WANG \\ Center for Fundamental Physics, University of Science and \\ Technology of China, Hefei, Anhui 230026, P. R. China
}

(Received November 4, 1994)

\begin{abstract}
In the present paper, considering oriented polymers interacting with a background tangent field $\mathbf{u}$, the Flory exponent $v$ is self-consistently calculated to be $v=3 /(d+2)$ for $d \leq 4$ and $v=1 / 2$ for $d \geq 4$ using the renormalization theory for turbulent transport. The results of this paper agree with that of Kamien [J. Phys. (Paris) I, 3, 1663 (1993)] by the dynamical renormalizationgroup method.

KEY WORDS Conformation of Polymer / Flory Exponent / Random Work / Fokker-Planck Equation / Renormalization-Group / Turbulent Transport / Renormalization Theory /
\end{abstract}

The problem of understanding the conformation of polymers is of both fundamental and practical importance. Flory ${ }^{1}$ predicted the wandering exponent $v$ defined by \langle| $\mathbf{r}(L)-$ $\left.\left.\mathbf{r}(0)\right|^{2}\right\rangle \sim L^{2 v}$ with the dimensional analysis. From the point of view that the polymer acts as a random walk in random environment, the Flory exponent can be interpreted as the exponent determined the long time behavior of the random walk. ${ }^{2}$ Considering oriented polymers interacting with a background tangent field $\mathbf{u}$, the Boltzmann weight $Z_{\mathbf{u}}$ is

$$
\begin{aligned}
Z_{u} & =N \int_{\mathbf{r}(0)=0}^{\mathbf{r}(s)=\mathbf{r}}[\mathrm{d} \mathbf{r}] \\
& \times \exp \left[-\frac{1}{4 D} \int_{0}^{s} \mathrm{~d} s^{\prime}\left(\frac{\mathrm{d} \mathbf{r}\left(s^{\prime}\right)}{\mathrm{d} s^{\prime}}-\mathbf{u}\left(\mathbf{r}\left(s^{\prime}\right), s^{\prime}\right)\right)^{2}\right]
\end{aligned}
$$

in which $N$ is the normalization constant, $D$ is the diffusion constant and the tangent field $\mathbf{u}(\mathbf{r}, s)$ is a function of both space and the monomer label $s$. The partition function $Z(\mathbf{r}, s)$ is

$$
Z(\mathbf{r}, s)=\int[\mathrm{d} \mathbf{u}] Z_{\mathbf{u}}(\mathbf{u}, s) P[\mathbf{u}]
$$

It is easy to find out that the probability distribution for $\mathbf{r}$ is just

$$
P(\mathbf{r}, s)=Z(\mathbf{r}, s) / \int \operatorname{dr} Z(\mathbf{r}, s)
$$

Viewing the Boltzmann weight $Z_{\mathbf{u}}$ as a quantum mechanical propagate in imaginary time, it can be shown that the Fokker-Planck equation for $Z_{u}$ is just the advection-diffusion equation if the polymers have no ends. ${ }^{3} \mathrm{By}$ the dynamical renormalization-group ( $R N G$ ) analysis of the equation, Kamien has selfconsistently calculated the wandering exponent $v=3 /(d+2)$ for $d \leq 4$ and $v=1 / 2$ for $d \geq 4$, which is exactly the Flory results.

The renormalization theory for eddy diffusivity in turbulent transport has been developed by Avellaneda and Majda. ${ }^{4-7}$ For the two dimensional anisotropic stratified flows, they have derived an exact represent formulas for the solution of the advection-diffusion equation through Fourier analysis and the FeynmanKac formula. ${ }^{4,5}$ It has been established that the renormalization problem for turbulent transport by isotropic homogeneous random fields in d-space dimension has exact the same phase diagram as that of the $2 \mathrm{D}$ anisotropic 
stratified flows. ${ }^{7}$ It is interesting to point out that the rigorous renormalization theory may provide an important nontrivial test problem for the capability of the RNG and renormalized perturbation theory method. The dynamical RNG analysis in an approximate method, which is to mimic the Wilson's first-order $\epsilon$ expansion from critical phenomena to predict the anomalous scaling exponents. The renormalization theory for turbulent transport is an exact mathematical method based upon the Faurier analysis and Feynman-Kac formula. Comparing with the results of the exact renormalization theory, Avellaneda and Majda ${ }^{6}$ have shown that the dynamical RNG analysis can predict the correct scaling exponents in some cases and in other cases it fails.

In this paper, with the renormalization theory, the Flory exponent is calculated and the same results as that of Kamien are obtained. It can be shown that the renormalization theory is useful for understanding the conformation of polymers.

\section{MODEL AND ANALYSIS}

If the polymers have no ends, the FokkerPlanck equation for $Z_{\mathrm{u}}$ is ${ }^{3}$

$$
\frac{\partial Z_{u}(\mathbf{r}, s)}{\partial s}+\mathbf{u}(\mathbf{r}, s) \cdot \nabla Z_{\mathbf{u}}(\mathbf{r}, s)=D \nabla^{2} Z_{\mathbf{u}}(\mathbf{r}, s)
$$

where the Gaussian random field $\mathbf{u}$ is specified by the two-point correlation:

$$
\begin{aligned}
& \left\langle u_{i}(\mathbf{k}, \omega) u_{i}\left(\mathbf{k}^{\prime}, \omega^{\prime}\right)\right\rangle=\frac{1}{(2 \pi)^{d / 2}} \frac{\lambda_{0}}{a_{0}} P_{i j}(\mathbf{k}) \\
& \times k^{2-d-\epsilon-\alpha} \phi\left(\frac{\omega}{a_{0} \kappa^{\alpha}}\right) \delta\left(\mathbf{k}+\mathbf{k}^{\prime}\right) \delta\left(\omega+\omega^{\prime}\right)
\end{aligned}
$$

in which $\mathbf{k}$ is the wave number which is the Fourier conjugate of $\mathbf{r}, d$ is the dimension of space, $\lambda_{0}, a_{0}, \epsilon$, and $\alpha$ are constants, and $P_{i j}(\mathbf{k})$ and $\phi(x)$ are given by

$$
P_{i j}(\mathbf{k})=\delta_{i j}-\frac{k_{i} k_{j}}{k^{2}}, \quad \phi(x)=\frac{1}{\pi\left(1+x^{2}\right)} .
$$

Table I. The five regions in the $(\epsilon, \alpha)$ plane with different behavior for the renormalization theory

\begin{tabular}{cll}
\hline Region & Regime for $\epsilon$ and $\alpha$ & $\begin{array}{c}\text { Scaling } \\
\text { exponents } z \\
\text { for time }\end{array}$ \\
\hline I & $\epsilon<\max (0,2-\alpha)$ & 2 \\
II & $2-\alpha<\epsilon \leq 4-2 \alpha$ & $4-\epsilon-\alpha$ \\
III & $4-2 \alpha \leq \epsilon<4$ for $\alpha \leq 1$ & $2-\epsilon / 2$ \\
& and $2<\epsilon<4$ for $\alpha \geq 1$ & \\
IV & $4-2 \alpha \leq \epsilon<2$ for $1 \leq \alpha<2$ & $2 \alpha /(\epsilon+2 \alpha-2)$ \\
V & $0<\epsilon<2$ and $\alpha \geq 2$ & $2 /(1+\epsilon / 2)$ \\
\hline
\end{tabular}

Avellaneda and Majda $^{4-7}$ have studied the problem of turbulent transport described by the eq 2 and 3. They have rigorously analyzed the renormalization problem for $2 \mathrm{D}$ anisotropic stratified flows with the statistics of the form $\mathbf{u}(\mathbf{x}, t)=\left[u\left(x_{2}, t\right), 0\right]$ with $\mathbf{x}=\left(x_{1}, x_{2}\right)$. From the view of the renormalization theory, $\epsilon$ and $\alpha$ should satisfied $\epsilon<4$ and $\alpha>0$. The values of the scaling exponent $z$ of time varying in the ranges $-\infty<\epsilon<4$ and $\alpha>0$ is shown in the phase diagram, which involves five regions with different scaling exponent $z$ of time (see Table I). Avellaneda and Majda have studied the problem of turbulent transport by isotopic homogeneous random fields in d-dimension with the regime $0<\alpha<1$ of the phase diagram. In the present work, we utilize the phase diagram to calculate the Flory exponent.

In the renormalization-group analysis of the eq 2 and 3, Kamien choose

$$
\epsilon=\Delta+2-d, \quad \alpha=\Delta
$$

so that

$$
\begin{aligned}
\left\langle u_{i}(\mathbf{k}, \omega) u_{j}\left(\mathbf{k}^{\prime}, \omega^{\prime}\right)\right\rangle= & \frac{\delta_{i j}-k_{i} k_{j} / k^{2}}{A \omega^{2}+B k^{2 \Delta}} \\
& \times \delta\left(\mathbf{k}+\mathbf{k}^{\prime}\right) \delta\left(\omega+\omega^{\prime}\right)
\end{aligned}
$$

Considering that the polymers acts as the random walk, there is the scaling relation for the position ${ }^{2}$ :

$$
\left\langle|\mathbf{r}(L)-\mathbf{r}(0)|^{2}\right\rangle \sim L^{2 / z}
$$


In order to obtain the self-consistent results, the scaling exponent $z$ for time should satisf $y^{3}$

$$
z=\Delta
$$

In the present work, we will evaluate the exponent $z$ and $\Delta$ with the help of the phase diagram and eq 4 and 7 . The detail analysis is given as follows:

(i) If the parameters $(\epsilon, \alpha)$ are located in region $\mathrm{I}(\epsilon<\max (0,2-\alpha))$, in which the mean field behavior occurs, the scaling exponent $z$ is

$$
z=2
$$

With the constraints of the eq 4 and 7 , we have

$$
\epsilon=4-d, \quad \alpha=2
$$

From eq 9 , it is easy to see that the parameters $(\epsilon, \alpha)$ are in region I only if

$$
d \geq 4
$$

(ii) If the parameters $(\epsilon, \alpha)$ are located in region II $(2-\alpha<\epsilon \leq 4-\alpha)$, the scaling exponent $z$ satisfies

$$
z=4-\epsilon-\alpha
$$

With the constraints of the eq 4 and 7 , we have

$$
\epsilon=\frac{8-2 d}{3}, \quad \alpha=\frac{d+2}{3}
$$

That is to say

$$
z=\Delta=\frac{d+2}{3}
$$

From eq 12 we can find out that the parameters $(\epsilon, \alpha)$ have to be located on the boundary line $\epsilon+2 \alpha=4$ for $d \leq 4$.

(iii) If the parameters $(\epsilon, \alpha)$ are located in region III $(4-2 \alpha \leq \epsilon<4$ for $2<\alpha$ and $2<\epsilon<4$ for $\alpha \geq 2$ ), the scaling exponent $z$ satisfies

$$
z=2-\frac{\epsilon}{2}
$$

With the constraints of the eq 4 and 7 , we have

$$
z=\Delta=\frac{d+2}{3}
$$

From the discussion (ii), we have the consequence that the parameters $(\epsilon, \alpha)$ have to be located on the boundary between the regions II, III, and IV for $d=1$ under the constraints of the eq 4 and 7.

(iv) If the parameters $(\epsilon, \alpha)$ are located in region IV $(4-2 \alpha<\epsilon<2$ for $1<z<2)$, the scaling exponent $z$ satisfies

$$
z=\frac{2 \alpha}{\epsilon+2 \alpha-2}
$$

With the constraints of the eq 4 and 7 , we have

$$
\epsilon=\frac{8-2 d}{3}, \quad \alpha=\Delta=\frac{d+2}{3}
$$

From eq 17, we have the consequence that the parameters $(\epsilon, \alpha)$ have to be located on the boundary between the regions II and IV for $d \leq 4$ under the constraints of the eq 4 and 7 .

(v) If the parameters $(\epsilon, \alpha)$ are located in region $V(0<\epsilon<2$ and $\alpha \geq 2)$, the scaling exponent $z$ satisfies

$$
z=\frac{2}{1+\epsilon / 2}
$$

With the constraints of the eq 4 and $7, \epsilon$ has to satisfy the relation:

$$
\epsilon<0
$$

So it is impossible that the parameters $(\epsilon, \alpha)$ are located in region $\mathrm{V}$ under the constrainst of the eq 4 and 7.

From the above discussions, we can find out that $z=\Delta=(d+2) / 3$ for $d \leq 4$ and $z=\Delta=2$ for $d \geq 4$, and $d=d_{\mathrm{c}}=4$ is the critical dimension, which has recently been obtained using the dynamical renormalization-group method by Kamien. ${ }^{3}$ From eq 7 , we can calculated the Flory exponent $v: v=3 /(d+2)$ for $d<4$, and $v=1 / 2$ for $d \geq 4$, which is exactly the Flory's result. 


\section{CONCLUSION}

In this paper, we utilize the renormalization theory to obtain the same results as that of Kamien, which will strengthen the reliability of his work. It is very interesting to find out that in the case of $d \leq 4$ the parameters $(\epsilon, \alpha)$ have to be located on the line $\epsilon+2 \alpha=4$, which is the boundary between the regions II, III, and IV. In the problem for turbulence, the Kolmogrov spectrum also sits at this boundary line with the values $\epsilon=8 / 3, \alpha=2 / 3$. Avellaneda and $\mathrm{Mijda}^{4,6,7}$ have shown that the boundary line $\epsilon+2 \alpha=4$ has some remarkable crossover properties. These properties might shed light on the problem why the RNG method and renormalization theory give the same results for calculating the Flory exponent.

\section{REFERENCES}

1. P. Flory, "Principles of Polymer Chemistry," Cornell University Press, Ithaca, New York, N. Y., 1971.

2. J. P. Bonchard and A. Georges, Phys. Rep., 195, 127 (1990).

3. R. D. Kamien, J. Phys. (Paris), I, 3, 1663 (1993).

4. M. Avellaneda and A. Majda, Commnn. Math. Phys., 131, 381 (1990).

5. M. Avellaneda and A. Majda, Commnn. Math. Phys., 138, 339 (1991).

6. M. Avellaneda and A. Majda, Phys. Fluids, A4, 41 (1992).

7. M. Avellaneda and A. Majda, Phys. Rev. Lett., 68, 3028 (1992). 